\title{
Novel tumor-suppressor gene epidermal growth factor-containing fibulin-like extracellular matrix protein 1 is epigenetically silenced and associated with invasion and metastasis in human gastric cancer
}

\author{
XIN-JIANG ZHU, JIAN LIU, XIAO-YANG XU, CHUN-DONG ZHANG and DONG-QIU DAI \\ Department of Gastrointestinal Surgery, The Fourth Affiliated Hospital, \\ China Medical University, Shenyang, Liaoning 110032, P.R. China \\ Received September 22, 2013; Accepted March 12, 2014
}

DOI: $10.3892 / \mathrm{mmr} .2014 .2135$

\begin{abstract}
The present study aimed to investigate the role of histone modification and DNA methylation in epidermal growth factor-containing fibulin-like extracellular matrix protein 1 (EFEMP1) silencing in gastric cancer (GC). In the present study, four GC cell lines, and 45 paired normal and GC tissue samples were used to assess EFEMP1 expression using quantitative polymerase chain reaction (PCR), and EFEMP1 gene methylation status was evaluated by methylation-specific PCR. The involvement of histone modification in GC cell lines was examined by a chromatin immunoprecipitation (ChIP) assay. The results demonstrated that EFEMP1 mRNA level and methylation status in the EFEMP1 promoter region was associated with tumor differentiation, depth of tumor invasion and lymph node metastasis. DNA methyltransferase inhibitor 5-aza-2'-deoxycytidine (DAC) rapidly reduced DNA methylation and histone $\mathrm{H} 3-\mathrm{K} 9$ trimethylation at the silenced loci and reactivated EFEMP1 expression. By contrast, the histone deacetylase inhibitor trichostatin A markedly increased histone $\mathrm{H} 3-\mathrm{K} 9$ acetylation. However, it had no effect on DNA methylation, histone $\mathrm{H} 3-\mathrm{K} 9$ trimethylation or gene expression. In conclusion, the results suggested that EFEMP1 may function as a tumor suppressor in GC. Aberrant DNA methylation and histone H3-K9 trimethylation of EFEMP1 may be responsible for its downregulation in GC, and thus have an important role in tumor invasion and metastasis.
\end{abstract}

Correspondence to: Professor Dong-Qiu Dai, Department of Gastrointestinal Surgery and Cancer Center, The Fourth Affiliated Hospital of China Medical University, 4 Chongshan East Road, Shenyang, Liaoning 110032, P.R. China

E-mail: daidq63@163.com

Key words: epidermal growth factor-containing fibrulin-like extracellular matrix protein 1, gastric carcinoma, DNA methylation, histone modification

\section{Introduction}

Gastric cancer (GC) is the second leading cause of cancer-associated mortality in males and the third leading cause in females worldwide (1). The majority of patients are diagnosed at an advanced stage and, thus, the overall treatment response is poor and the five-year survival rate is low. Understanding the molecular pathophysiology of GC is essential for determining methods to effectively inhibit tumor progression.

Recent studies have demonstrated that epigenetic mechanisms are closely associated with the development, progression and metastasis of GC. These epigenetic changes include DNA methylation, histone modifications and miRNA expression (2). Aberrant DNA methylation and histone modification occurs in the promoter $\mathrm{CpG}$ island of tumor suppressor genes (TSGs) where DNA is transcribed into RNA (3). EFEMP1 has antiangiogenic activity via suppression of endothelial cell sprouting (4), suggesting that it has a critical role in cancer development. However, the association between EFEMP1 deregulation and cancer remains a matter of debate. In support of a possible tumor-suppressive role, downregulation of EFEMP1 gene and/or EFEMP1 promoter methylation occurs in lung, liver, prostate, breast and nasopharyngeal carcinoma (5-9). In addition, EFEMP1 may have a potential cancer-promoting function in cervical cancer (10), breast carcinoma (11) and pancreatic cancer (12). These studies have demonstrated that EFEMP1 has contrasting roles in cancer, depending on the tissue in which it is expressed. The 5'-end of the EFEMP1 gene contains numerous $\mathrm{CpG}$ islands, suggesting that its expression may be controlled by DNA methylation and histone modification. Hypermethylation of the EFEMP1 promoter has been reported in various human malignancies. This finding prompted us to examine whether epigenetic silencing of EFEMP1 was involved in carcinogenesis.

To the best of our knowledge, the role of EFEMP1 in GC has not been examined to date. The present study aimed to verify whether decreased EFEMP1 expression in GC is associated with DNA methylation and histone modification. Four GC cell lines were used to examine EFEMP1 mRNA expression, DNA methylation and histone modification of EFEMP1. In addition, 45 GC specimens and 45 corresponding 
non-malignant gastric tissues were recruited to observe the mRNA expression and DNA methylation of EFEMP1 as well as its clinical significance.

\section{Materials and methods}

Cell culture and treatment with 5-aza-2'-deoxycytidine (DAC) and trichostatin A (TSA). Four human GC cell lines, MKN45, SGC7901, BGC823 and AGS were obtained from the Institute of Biochemistry and Cell Biology, Chinese Academy of Sciences (Shanghai, China). One immortalized normal gastric cell line, GES1, was obtained from the Oncology Institute of China Medical University (Shenyang, China). These cells were cultured in RPMI-1640 medium (Gibco-BRL, Grand Island, NY, USA) supplemented with $10 \%$ fetal bovine serum (Gibco-BRL) and incubated at $37^{\circ} \mathrm{C}$ in a humidified $5 \% \mathrm{CO}_{2}$ atmosphere. MKN45, SGC7901, BGC823 and AGS cells were incubated for $24 \mathrm{~h}$ prior to treatment as follows: (i) DAC group, $5 \mu \mathrm{M}$ DAC (Sigma, St. Louis, MO, USA) was added and cells were incubated for three days and the medium containing DAC was refreshed every day; (ii) TSA group, $0.3 \mu \mathrm{M}$ TSA (Sigma) for $24 \mathrm{~h}$; (iii) DAC + TSA group, $5 \mu \mathrm{M}$ DAC for $48 \mathrm{~h}$ followed by $0.3 \mu \mathrm{M}$ TSA for an additional $24 \mathrm{~h}$; (iv) control group, control cells of the same batch were incubated without DAC or TSA, with replacement of fresh medium on the same schedule as that used for the drug-treated cells. The time, dose and sequence of DAC and/or TSA are based on those of previous studies $(13,14)$.

Tissue samples. Human GC samples were collected from 45 patients who underwent a gastrectomy at the Department of Surgery, China Medical University (Shenyang, China) between January 2009 and June 2011. All GC cases were pathologically confirmed. Non-malignant gastric tissues that were at least $5 \mathrm{~cm}$ away from the tumor, were obtained from the patients.

Wound healing assay. The cells were plated in 6-well plates and maintained in RPMI-1640 medium containing 10\% fetal calf serum. A wound was created in the center of the cell monolayer by a sterile plastic pipette tip. The cells were allowed to migrate for $24 \mathrm{~h}$. The images were captured at 0,12 and $24 \mathrm{~h}$ following wounding to assess the ability of the cells to migrate into the wound area using an inverted microscope (IX-71; Olympus, Tokyo, Japan).

Matrigel invasion assay. Approximately $5 \times 10^{4}$ cells cultured in $200 \mu \mathrm{l}$ serum-free RPMI-1640 medium were seeded onto Matrigel-coated 8- $\mu \mathrm{m}$ pore size Transwell filters (Corning Life Sciences, Corning, NY, USA) in the upper chambers. A total of $500 \mu \mathrm{l}$ RPMI-1640 containing 10\% fetal calf serum was added to the lower chambers as a chemoattractant. The cells were incubated at $37^{\circ} \mathrm{C}$ in a humidified $5 \% \mathrm{CO}_{2}$ atmosphere for $24 \mathrm{~h}$. The cells that had successfully invaded through the inserts were fixed in $4 \%$ paraformaldehyde for $30 \mathrm{~min}$ and stained with methylrosanilinium chloride. The invaded cells were counted from five pre-selected microscopic fields of view at a magnification of $x 200$. The results of the Matrigel invasion assay were obtained from three independent experiments.

Cell counting kit-8 (CCK-8) assay. The cell proliferative ability was evaluated by a CCK-8 assay (C0037; Beyotime Institute of Biotechnology, Shanghai, China). The cells were seeded in

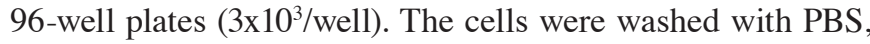
and the medium was replaced with fresh medium containing DAC ( $5 \mu \mathrm{mol} / \mathrm{l})$ and/or TSA (300 nmol/l). Following culture, the CCK-8 solution (10 $\mu \mathrm{l} / 100 \mu \mathrm{l}$ medium) was added to each well and the cells were incubated for $1 \mathrm{~h}$ at $37^{\circ} \mathrm{C}$. The absorbance was measured at $450 \mathrm{~nm}$ using a Synergy 2 Multi-Mode Microplate reader (BioTek, Winooski, VT, USA). The GC cells cultured in RPMI-1640 without DAC or TSA were used as the controls. The assay was conducted in five replicate wells for each sample and three parallel experiments were performed.

$R N A$ extraction and quantitative polymerase chain reaction $(q P C R)$. The total RNA was extracted from GC cells, human $\mathrm{GC}$ tissues and the corresponding non-cancerous tissues from the same patients. Extraction was performed using TRIzol reagent (Invitrogen Life Technologies, Carlsbad, CA, USA) according to the manufacturer's instructions and reversely transcribed into cDNA using an Expand Reverse Transcriptase kit (Takara, Dalian, China). The expression of EFEMP1 mRNA was detected using qPCR with the following program: $95^{\circ} \mathrm{C}$ for $30 \mathrm{sec}, 35$ cycles of $95^{\circ} \mathrm{C}$ for $5 \mathrm{sec}$ and $60^{\circ} \mathrm{C}$ for $30 \mathrm{sec}$. The reaction mixture contained $12.5 \mu \mathrm{l} \mathrm{SYBR}$ Green (Takara), $1 \mu \mathrm{l}$ each primer, $2 \mu \mathrm{l}$ cDNA and $8.5 \mu 1$ diethylpyrocarbonate (DEPC)-treated water. The primers used were as follows: Sense: 5'-CGCCAGCACATTGTGAATGAC-3' and antisense: 5'-TTTGAGTTGCACTCCACCACG-3' for EFEMP1; and sense: 5'-CATGAGAAGTATGACAACAGCCT-3' and antisense: 5'-AGTCCTTCCACGATACCAAAGT-3' for GADPH. The negative control used DEPC-treated water to replace the cDNA templates for every PCR. The EFEMP1 level was expressed as $\mathrm{Ct}$ following normalization to the levels of GAPDH mRNA.

Chromatin immunoprecipitation (ChIP) assay. ChIP assays were performed as described previously (15) to measure the levels of histone methylation and acetylation at EFEMP1 promoter regions in GES1, MKN45, SGC7901, BGC823 and AGS cell lines. The cells were fixed to crosslink the nuclear protein to DNA by adding formaldehyde, resuspended in lysis buffer and sonicated to generate $\sim 500-1,000$ bp DNA fragments using a sonicator (Shanghai Bilon Instrument Co., Ltd., Shanghai, China). The main soluble chromatin fraction was immunoprecipitated using antibodies against Lys-9 trimethylated histone H3 antibody (05-1242; Millipore, Billerica, MA, USA) or Lys-9 acetylated histone H3 (07-352; Millipore). The remaining soluble fraction was incubated with normal rabbit IgG (negative control). In addition, 1/100 of the soluble fraction collected prior to adding the antibody was used as an internal control for the quantity of input DNA. The crosslinking between DNA and proteins was reversed by heating the samples at $65^{\circ} \mathrm{C}$ for $4 \mathrm{~h}$, followed by proteinase $\mathrm{K}$ digestion. The DNA was then extracted with phenol/chloroform. A total of $2 \mu 1$ of immunoprecipitated DNA, DNA input control and negative control were used for PCR. The following primer set for PCR were designed to amplify the overlapping fragments of $131 \mathrm{bp}$ along the EFEMP1 promoter: Sense: 5'-ATCCCTTGATGGACACTT-3' and antisense: 5'-TCTCATTTCTGGGTATTTACT-3'. PCR products were subjected to $2.5 \%$ agarose gel electrophoresis 
at $120 \mathrm{~V}$ for $40 \mathrm{~min}$ and quantified using the Fluor Chen 2.0 system (Bio-Rad, Hercules, CA, USA). The levels of histone modification in each immunoprecipitation were calculated by quantifying the intensity of the PCR product in the immunoprecipitated DNA vs. the DNA input control. The ChIP experiments were repeated three times.

Methylation-specific PCR (MSP). Genomic DNA $(2 \mu \mathrm{g})$ was treated with $\mathrm{NaOH}(2 \mathrm{M})$ at $42^{\circ} \mathrm{C}$ for $20 \mathrm{~min}$. Following denaturation, the DNA was incubated with hydroquinone and sodium bisulfate at $54^{\circ} \mathrm{C}$ for $16 \mathrm{~h}$ in the dark. The DNA was purified using a DNA clean up kit (Promega Corporation, Madison, WI, USA), followed by incubation with $3 \mathrm{M} \mathrm{NaOH}$ at $37^{\circ} \mathrm{C}$ for $15 \mathrm{~min}$, and precipitation with ammonium acetate and $100 \%$ ethanol at $-20^{\circ} \mathrm{C}$ overnight. The following day, DNA was washed with $70 \%$ ethanol and dissolved in $15 \mu \mathrm{l}$ Tris-EDTA buffer. The primers used for MSP were located in the promoter region of the EFEMP1 gene. The CpG map of the EFEMP1 promoter and the location of primers used in the present study were based on a previous investigation (16). The primers for the methylated EFEMP1 CpG island were: Sense: 5'-TTTTTTCGTAGGGCGTTTTTTATC-3' and antisense: 5'-TTATAATCTACGATCGAACCTCGATT-3'. The primers for the unmethylated EFEMP1 CpG islands were sense: 5'-GCGGATTGTTTCGGGAGATC-3' and antisense: 5'-CAAAAAACGAAAATAAAACGACGAC-3'. Peripheral blood cell DNA from healthy adults treated with SssI methyltransferase (New England Biolabs, Ipswich, MA, USA) and untreated DNA were used as the positive and negative controls, respectively. Water blanks were used as a negative control. PCR products were separated by electrophoresis on $2 \%$ agarose gels and quantified using the Fluor Chen 2.0 system.

Statistical analysis. All statistical analyses were performed using SPSS version 17.0 (SPSS, Inc., Chicago, IL, USA). The $\chi^{2}$ and Fisher's exact tests were used for categorical variables, and Student's t-test or one-way analysis of variance for continuous variables. The relative mRNA expression levels (EFEMP1/GAPDH) were calculated from the quantified data. Data are expressed as the mean \pm standard deviation. $\mathrm{P}<0.05$ was considered to indicate a statistically significant difference.

\section{Results}

Effect of DAC and TSA on migration, invasion and proliferation of MKN45 cells in vitro. To investigate the inhibitory effect of DAC and TSA on migration of MKN45 cells, a wound-healing assay was performed. At $24 \mathrm{~h}$ following establishing the wound, the control group achieved almost complete wound closure $(80.00 \pm 0.9129 \%)$, compared with $64.75 \pm 0.8539 \%$ in the TSA group and only $52.25 \pm 0.6292$ and $53.25 \pm 1.109 \%$ in the DAC and DAC + TSA groups, respectively. These results demonstrated that DAC reduced cell migration significantly. Furthermore, TSA had a moderate effect on the migration of MKN45 cells. Combined treatment with the two agents reduced cell migration similar to that with DAC ( $\mathrm{P}<0.01$; Fig. 1A and B).

In order to examine whether DAC and TSA regulate GC invasion, the invasive capability of MKN45 cells was investigated using a Matrigel invasion assay. The numbers of cells penetrating Matrigel and adhering to the membrane in the control, DAC, TSA and DAC + TSA groups was 217.4 \pm 3.723 , $108 \pm 3.033,146.2 \pm 3.455$ and $49.8 \pm 1.158$, respectively. A decrease in cell numbers in the DAC, TSA and DAC + TSA groups was observed, compared with the control group $(\mathrm{P}<0.01$; Fig. $1 \mathrm{C}$ and $\mathrm{D})$.

To determine the effect of DAC and TSA on MKN45 cell growth in vitro, following exposure to DAC ( $5 \mu \mathrm{mol} / \mathrm{l})$ and/or TSA (300 nmol/l) for 24,48 and $72 \mathrm{~h}$, the cell proliferation was analyzed by a CCK- 8 assay. The results indicated that MKN45 cells treated with DAC and combined DAC and TSA exhibited growth retardation at 48 and $72 \mathrm{~h}$, compared with the controls. TSA only had a significant inhibitory effect on MKN45 cell proliferation following $72 \mathrm{~h}$. However, there was no significant difference in the cell proliferation activity between the treated cells and control groups with 24 h exposure. (Fig. 1E).

EFEMP1 expression is downregulated in GC cells and tissues. qPCR was performed to assess the mRNA expression of EFEMP1, which was substantially downregulated in MKN45 $(0.323 \pm 0.002)$, AGS $(0.407 \pm 0.008)$, BGC823 (0.454 \pm 0.024$)$ and SGC7901 $(0.806 \pm 0.018)$ cells compared with the normal mucosa line, GES1 (1-fold as the control; P<0.05; Fig. 2A). In order to examine whether epigenetic agents were able to reverse EFEMP1 silencing in GC cells, the cells were treated with the DNA methyltransferase (DNMT) inhibitor DAC, the histone deacetylase (HDAC) inhibitor TSA and combined treatment with the two agents. qPCR demonstrated that DAC and TSA had different effects on EFEMP1 expression in the GC cell lines. In the cells with low expression of EFEMP1 (MKN45, AGS and BGC823), DAC alone restored EFEMP1 expression. TSA had no effect on EFEMP1 expression. Combined treatment restored EFEMP1 expression similar to that with DAC. In the EFEMP1-positive cell lines (SGC7901), treatment with DAC and TSA, alone or in combination, had no significant effect on the expression of EFEMP1 (Fig. 2B).

EFEMP1 expression was analyzed in 45 paired GC specimens and corresponding normal tissues by qPCR. It was identified that EFEMP1 mRNA expression was significantly lower in the GC tissues than in their corresponding normal tissues $(0.334 \pm 0.011$ vs. $0.557 \pm 0.015$; $\mathrm{P}<0.0001$; Fig. 3A; Table I). Furthermore, the correlation between EFEMP1 mRNA expression and the clinicopathological factors of GC were examined. The EFEMP1 mRNA expression level was associated with tumor differentiation, depth of tumor invasion and lymph node metastasis (Fig. 3B; Table I).

Low expression of EFEMP1 is associated with DNA methylation. To identify whether DNA methylation was responsible for the downregulation of EFEMP1 expression, the DNA methylation status of EFEMP1 in GC cell lines and GES1 cells was assessed using MSP. The EFEMP1-positive cell lines (GES1 and SGC7901) demonstrated unmethylated bands (neither allele was methylated), which was in agreement with the observed high levels of EFEMP1 expression. By contrast, the MKN45 and AGS cells only demonstrated methylated bands (hypermethylated, both alleles methylated), BGC823 cells exhibited methylated and unmethylated bands (partially methylated, only one allele methylated), which was in agreement with the observed low levels of EFEMP1 
A

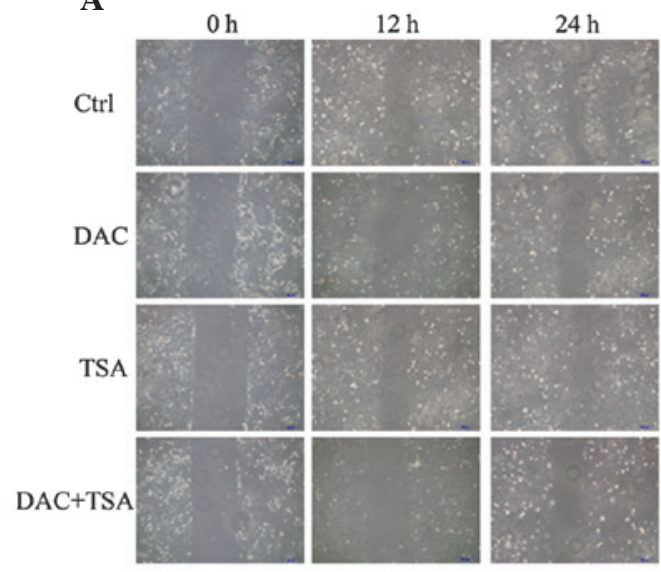

B

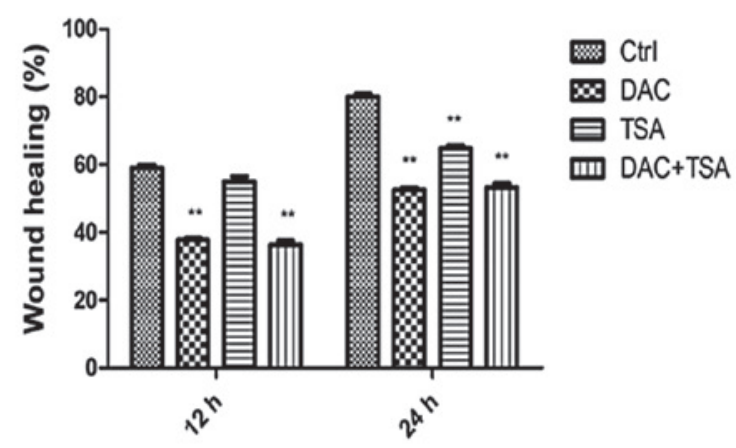

C

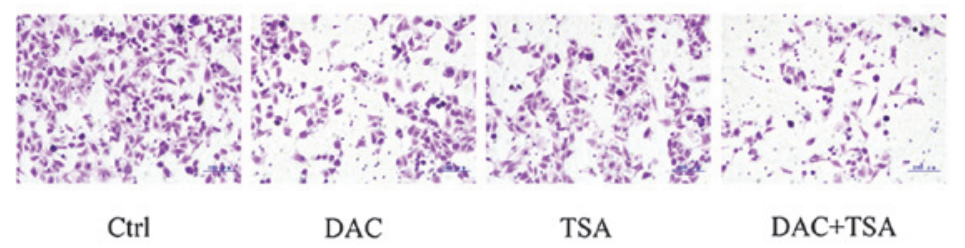

$\mathbf{E}$

\section{D}

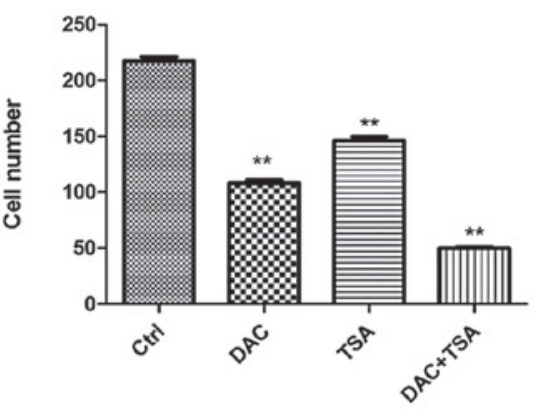

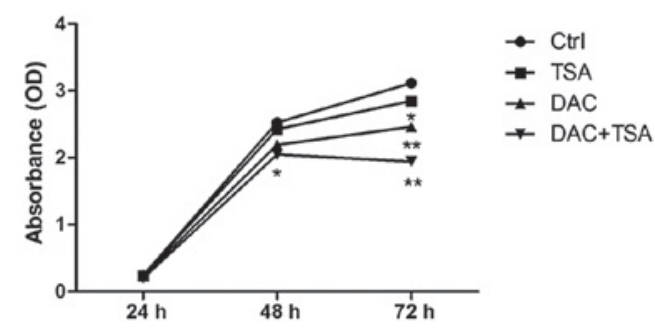

Figure 1. Effect of DAC and/or TSA on MKN45 cell migration, invasion and proliferation. (A) Wound healing assay of the MKN45 cells. The images were captured at 0,12 and $24 \mathrm{~h}$ following the wound incision (magnification, x100). (B) The percentage of wound closure was measured in at least three randomly selected regions (mean $\pm \mathrm{SD}$ ). (C) The invasion ability of MKN45 cells was observed by Matrigel invasion assay following treatment with DAC and/or TSA. Representative images of the treated and untreated cells are presented (magnification, x200). (D) The columns indicate the number of cells invading at the $24 \mathrm{~h}$ time point. The values represent the mean \pm SD. (E) MKN45 cells were treated with DAC and/or TSA and proliferation was estimated at $24 \mathrm{~h}$ intervals up to $72 \mathrm{~h}$ using a cell counting kit- 8 assay. Data are presented as the mean \pm SD. ${ }^{*} \mathrm{P}<0.05,{ }^{* *} \mathrm{P}<0.01$, compared with the Ctrl. SD, standard deviation; DAC, 5-aza-2'-deoxycytidine; TSA, trichostatin A; Ctrl, control.

A

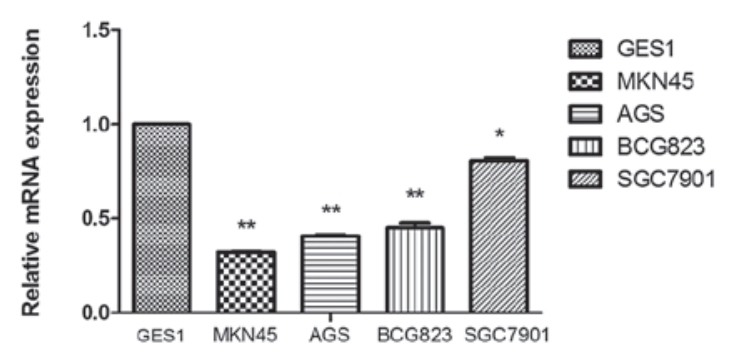

B

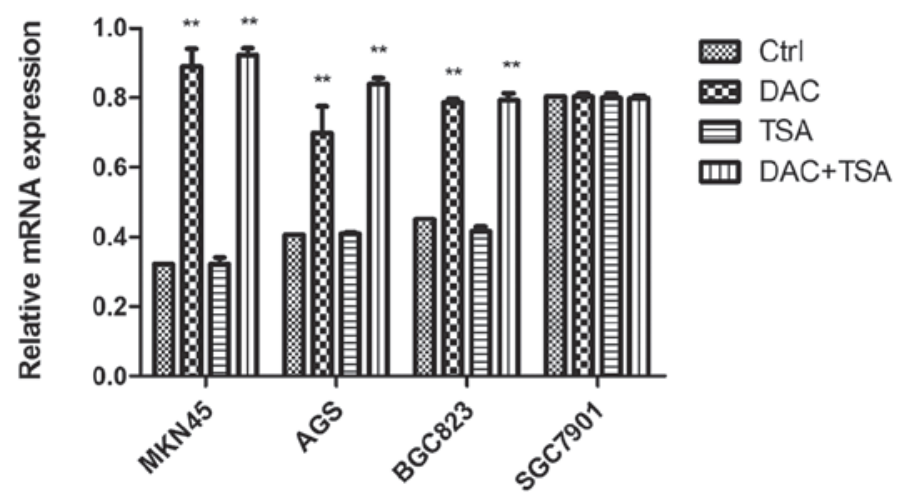

Figure 2. qPCR analysis of EFEMP1 mRNA expression in human GC cells. (A) Expression of EFEMP1 in GES1 cells was higher than in the four different gastric cancer cell lines and was lowest in MKN45. (B) qPCR analysis of EFEMP1 mRNA expression prior to and following treatment of human gastric cancer cells with DAC, TSA or DAC+TSA. "P $<0.05,{ }^{* *} \mathrm{P}<0.01$, compared with the Ctrl. EFEMP1, epidermal growth factor-containing fibulin-like extracellular matrix protein 1; qPCR, quantitative polymerase chain reaction; DAC, 5-aza-2'-deoxycytidine; TSA, trichostatin A; GC, gastric cancer; Ctrl, control. 
Table I. Correlation between the clinicopathological features and EFEMP1 mRNA expression in 45 GC patients.

\begin{tabular}{|c|c|c|c|}
\hline Variable & Patients (n) & $\begin{array}{l}\text { EFEMP1 mRNA expression } \\
\text { relative to GAPDH }\end{array}$ & P-value \\
\hline Normal & 45 & $0.557 \pm 0.015$ & $<0.01^{\mathrm{a}}$ \\
\hline Tumor & 45 & $0.334 \pm 0.011$ & \\
\hline \multicolumn{4}{|l|}{ Age (years) } \\
\hline$<65$ & 23 & $0.331 \pm 0.015$ & 0.828 \\
\hline$\geq 65$ & 22 & $0.336 \pm 0.016$ & \\
\hline \multicolumn{4}{|l|}{ Gender } \\
\hline Male & 27 & $0.325 \pm 0.013$ & 0.499 \\
\hline Female & 18 & $0.340 \pm 0.019$ & \\
\hline \multicolumn{4}{|c|}{ Tumor differentiation } \\
\hline Well/moderate & 26 & $0.358 \pm 0.014$ & $<0.05^{\mathrm{a}}$ \\
\hline Poor & 19 & $0.300 \pm 0.015$ & \\
\hline \multicolumn{4}{|l|}{ Invasion depth } \\
\hline $\mathrm{T} 1+\mathrm{T} 2$ & 23 & $0.368 \pm 0.014$ & $<0.01^{\mathrm{a}}$ \\
\hline $\mathrm{T} 3+\mathrm{T} 4$ & 22 & $0.300 \pm 0.013$ & \\
\hline \multicolumn{4}{|l|}{ Tumor location } \\
\hline Upper + middle & 16 & $0.334 \pm 0.018$ & 0.803 \\
\hline Lower & 29 & $0.331 \pm 0.013$ & \\
\hline \multicolumn{4}{|l|}{ Size $(\mathrm{cm})$} \\
\hline$<3$ & 28 & $0.332 \pm 0.013$ & 0.868 \\
\hline$\geq 3$ & 17 & $0.336 \pm 0.020$ & \\
\hline \multicolumn{4}{|c|}{ Lymph node metastasis } \\
\hline No & 23 & $0.374 \pm 0.014$ & $<0.01^{\mathrm{a}}$ \\
\hline Yes & 22 & $0.291 \pm 0.011$ & \\
\hline
\end{tabular}

${ }^{a} \mathrm{P}<0.05$. GC, gastric cancer; EFEMP1, epidermal growth factor-containing fibulin-like extracellular matrix protein 1.

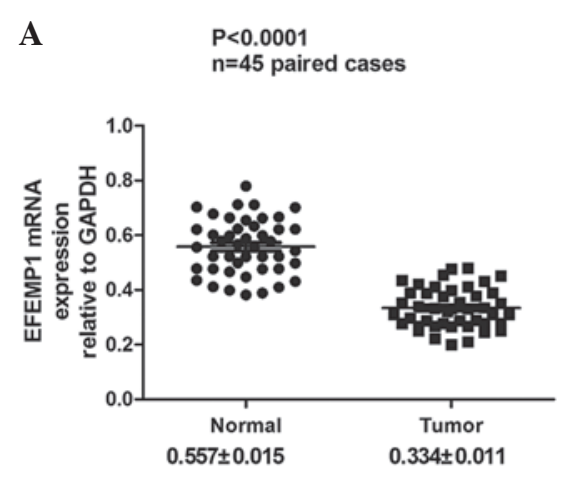

B

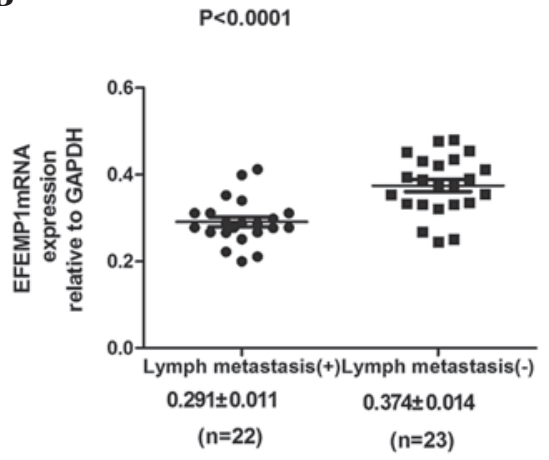

C

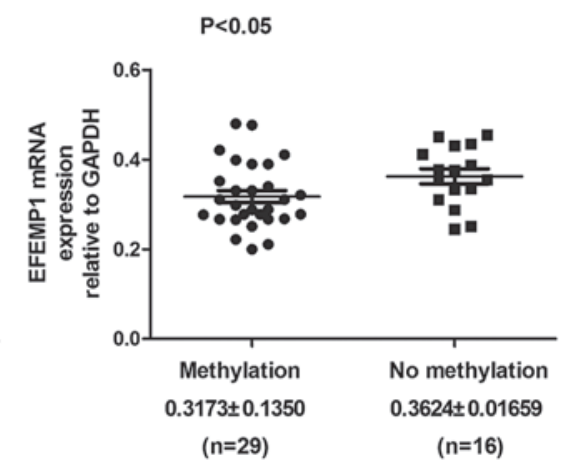

Figure 3. qPCR analysis of EFEMP1 mRNA expression in human GC tissues. (A) EFEMP1 mRNA expression in gastric cancer tissues and corresponding non-tumor tissues. (B) EFEMP1 mRNA expression level in patients with lymph node metastasis and those in tumors without lymph node metastasis. (C) Association between EFEMP1 methylation and downregulation of EFEMP1 mRNA. EFEMP1, epidermal growth factor-containing fibulin-like extracellular matrix protein 1; qPCR, quantitative polymerase chain reaction; GC, gastric cancer.

expression (Fig. 4A). In hypermethylated MKN45, AGS and BGC823 cells, treatment with DAC resulted in DNA demethylation. TSA had no effect on DNA methylation, and treatment with the two agents had no additional effect on DNA demethylation beyond that produced by treatment with DAC alone. In unmethylated SGC7901 cells, treatment with DAC, TSA or the two agents had no significant effect on DNA methylation (Fig. 4B).

EFEMP1 methylation in gastric specimens, including 45 tumor and 45 corresponding non-malignant gastric tissues, was then examined by MSP. Hypermethylation of the EFEMP1 gene was detected in $29(64.44 \%)$ of the 45 primary gastric 
Table II. Methylation status of EFEMP1 between T and N.

\begin{tabular}{lccc}
\hline Group & Case & Methylation $(\%)$ & No methylation $(\%)$ \\
\hline $\mathrm{T}$ & 45 & $29(64.44)$ & $16(35.56)$ \\
$\mathrm{N}$ & 45 & $11(24.44)$ & $34(75.56)$
\end{tabular}

${ }^{\mathrm{a}} \mathrm{P}<0.05$. T, gastric tissues; $\mathrm{N}$, non-malignant gastric tissues; EFEMP1, epidermal growth factor-containing fibulin-like extracellular matrix protein 1 .

Table III. Clinicopathological parameters of GC samples and EFEMP1 methylation.

\begin{tabular}{|c|c|c|c|c|}
\hline \multirow[b]{2}{*}{ Variable } & \multirow[b]{2}{*}{ Patients (n) } & \multicolumn{2}{|c|}{ EFEMP1 methylation } & \multirow[b]{2}{*}{ P-value } \\
\hline & & $\mathrm{M}(\%)$ & $\mathrm{U}(\%)$ & \\
\hline \multicolumn{5}{|l|}{ Age (years) } \\
\hline$<65$ & 22 & $13(59.1)$ & $9(40.9)$ & \multirow[t]{2}{*}{0.104} \\
\hline$\geq 65$ & 23 & $16(69.6)$ & $7(30.4)$ & \\
\hline \multicolumn{5}{|l|}{ Gender } \\
\hline Male & 27 & $17(62.9)$ & $10(37.1)$ & \multirow[t]{2}{*}{0.553} \\
\hline Female & 18 & $12(66.7)$ & $6(33.3)$ & \\
\hline \multicolumn{5}{|c|}{ Tumor differentiation } \\
\hline Well/moderate & 26 & $11(42.3)$ & $15(57.7)$ & \multirow[t]{2}{*}{$<0.01^{\mathrm{a}}$} \\
\hline Poor & 19 & $18(94.7)$ & $1(5.3)$ & \\
\hline \multicolumn{5}{|l|}{ Invasion depth } \\
\hline $\mathrm{T} 1+\mathrm{T} 2$ & 23 & $10(43.4)$ & $13(56.6)$ & \multirow[t]{2}{*}{$<0.01^{\mathrm{a}}$} \\
\hline $\mathrm{T} 3+\mathrm{T} 4$ & 22 & $19(86.3)$ & $3(13.7)$ & \\
\hline \multicolumn{5}{|l|}{ Tumor location } \\
\hline Upper + middle & 16 & $9(56.3)$ & $7(43.7)$ & \multirow[t]{2}{*}{0.058} \\
\hline Lower & 29 & $20(69.0)$ & $9(31.0)$ & \\
\hline \multicolumn{5}{|l|}{ Size $(\mathrm{cm})$} \\
\hline$<3$ & 28 & $19(67.9)$ & $9(32.1)$ & \multirow[t]{2}{*}{0.186} \\
\hline$\geq 3$ & 17 & $10(58.8)$ & $7(41.2)$ & \\
\hline \multicolumn{5}{|c|}{ Lymph node metastasis } \\
\hline No & 23 & $15(65.2)$ & $8(34.8)$ & \multirow[t]{2}{*}{0.883} \\
\hline Yes & 22 & $14(63.6)$ & $8(36.4)$ & \\
\hline
\end{tabular}

${ }^{\mathrm{a}} \mathrm{P}<0.05$. M, methylated and partially methylated cases; U, unmethylated cases; GC, gastric cancer; EFEMP1, epidermal growth factor-containing fibulin-like extracellular matrix protein 1.

carcinomas, while methylation in the non-malignant gastric tissue was only identified in 11 cases $(24.44 \%)$. No methylation was observed in $16(35.56 \%)$ primary GC tissues and 34 (75.56\%) non-malignant gastric tissues (Fig. 4C). The difference in methylation status of EFEMP1 between the primary GC and non-malignant gastric tissue specimens was significant $(\mathrm{P}<0.001$; Table II). It was also identified that EFEMP1 mRNA expression was significantly lower in the GC tissues with DNA methylation of EFEMP1 than that in GC tissues without DNA methylation of EFEMP1 (0.3173 \pm 0.1350 vs. $0.3624 \pm 0.01659$; $\mathrm{P}<0.05$; Fig. 3C). In addition, the correlation between gastric tumor EFEMP1 methylation status and the clinicopathological features of the patients was analyzed. The DNA methylation status of EFEMP1 was associated with tumor invasion depth and differentiation, but there was no correlation with the other clinicopathological features, including age, gender and tumor location (Table III).

Abnormal histone modification is associated with EFEMPI gene silencing in GC cell lines. The results demonstrated that EFEMP1 was downregulated in GC. However, the mechanism by which the expression of EFEMP1 was inhibited remains unknown. To elucidate whether abnormal histone modification was associated with the loss of EFEMP1, the basic levels of $\mathrm{H} 3-\mathrm{K} 9$ trimethylation and $\mathrm{H} 3-\mathrm{K} 9$ acetylation in the EFEMP1 promoters were compared in the GES1 and GC cells 

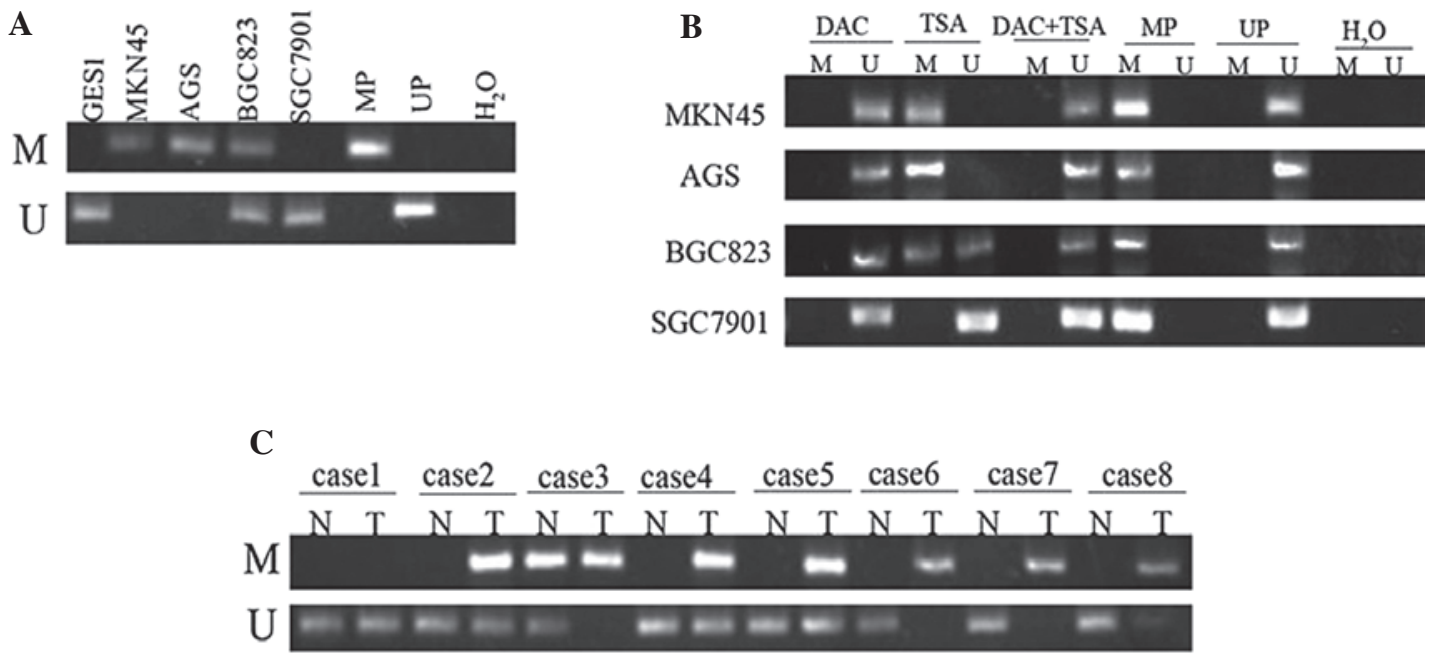

Figure 4. MSP analysis of DNA methylation at the EFEMP1 promoter region in human GC cells and tissues. (A) EFEMP1 was hypermethylated in MKN45 and AGS, and partially methylated in BGC823, but not methylated in GES1 and SGC7901 cells. (B) MSP analysis of DNA methylation at the EFEMP1 promoter region prior to and following treatment with DAC, TSA or DAC+TSA. (C) DNA methylation of EFEMP1 in GC specimens and corresponding non-malignant gastric tissues. Lane $\mathrm{M}$ indicates the presence of methylated alleles; lane $\mathrm{U}$ indicates the presence of unmethylated alleles. At least three independent experiments were performed with similar results. U, unmethylated; M, methylated; UP, non-methylation positive control; MP, methylation positive control; N, non-malignant gastric tissue; T, tumor specimens; MSP, methylation-specific PCR; EFEMP1, epidermal growth factor-containing fibulin-like extracellular matrix protein 1; DAC, 5-aza-2'-deoxycytidine; TSA, trichostatin A; GC, gastric cancer.
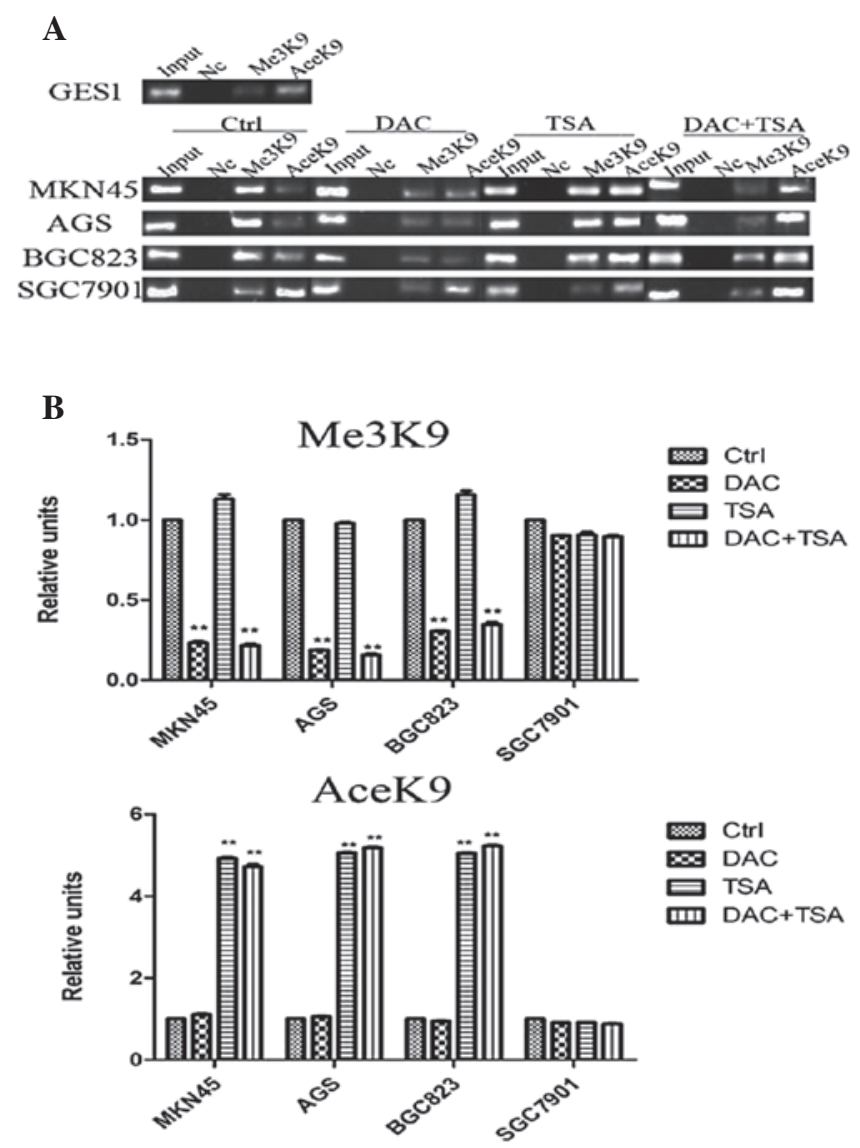

Figure 5. ChIP analysis of histone H3-K9 modification in the EFEMP1 promoter region in GES1 and four GC cell lines. Three independent ChIP assays were performed using an antibody that recognizes H3-K9 trimethylation and $\mathrm{H} 3-\mathrm{K} 9$ acetylation at the EFEMP1 promoter region. (A) Representative graphs of the PCR assay. (B) Average precipitated DNA/input DNA ratios on the $\mathrm{y}$-axis represent the relative values of $\mathrm{H} 3-\mathrm{K} 9$ trimethylation and $\mathrm{H} 3-\mathrm{K} 9$ acetylation. ${ }^{* *} \mathrm{P}<0.01$, compared with the Ctrl. ChIP, chromatin immunoprecipitation; GC, gastric cancer; EFEMP1, epidermal growth factor-containing fibulin-like extracellular matrix protein 1; DAC, 5-aza-2'-deoxycytidine; TSA, trichostatin A; PCR, polymerase chain reaction; Ctrl, control. with different EFEMP1 expression, using ChIP. As revealed in Fig. 4A, in the EFEMP1-positive cell lines (GES1 and SGC7901), H3-K9 trimethylation of the promoter regions was minimal. H3-K9 trimethylation levels in the EFEMP1 gene promoter were higher in cells with low expression of EFEMP1 (MKN45, AGS and BGC823). By contrast, H3-K9 acetylation at the EFEMP1 promoter region was significantly higher in GES1 and SGC7901 cells than in the MKN45, AGS and BGC823 cells.

To clarify whether epigenetic agents may affect epigenetic modifications, the GC cells were treated with DAC and TSA. It was identified that the levels of H3-K9 trimethylation in the EFEMP1 promoter in MKN45, AGS and BGC823 cells was decreased significantly following treatment with DAC, and TSA marginally reduced histone $\mathrm{H} 3-\mathrm{K} 9$ trimethylation. The effects of combined treatment with DAC and TSA on H3-K9 trimethylation were similar to those of DAC alone. H3-K9 acetylation at EFEMP1 promoter regions was analyzed using ChIP assays to determine whether DAC and TSA may affect H3-K9 acetylation in GC cells. In MKN45, AGS and BGC823 cells, treatment with TSA alone significantly increased H3-K9 acetylation, but DAC alone had no effect on H3-K9 acetylation. The effects of combined treatment with DAC and TSA on H3-K9 acetylation were similar to that of TSA alone. Treatment with both DAC and TSA had no significant effect on H3-K9 trimethylation and H3-K9 acetylation in SGC7901 cells, in which EFEMP1 was expressed (Fig. 5A and B).

\section{Discussion}

Epigenetics attempts to explain how heritable changes in gene expression occur without altering nucleotide sequence, and how epigenetic alterations have an important role in silencing TSGs (17). In GC, a growing number of TSGs have been identified as undergoing aberrant methylation. When DNA is methylated in the promoter region of the genes, where 
transcription is initiated, they are typically inactivated and silenced (18-20). Modification of the histone tail is another epigenetic regulatory mechanism. The acetylation of lysine residues on histone $\mathrm{H} 3-\mathrm{K} 9$ leads to the formation of an open chromatin structure and allows regulatory factors to access the chromatin, which is an active marker, but methylation of histone H3-K9 is a marker of gene inactivity (21). Increasing evidence now indicates that DNA methylation and histone modifications appear to be linked to each other. DNA methylation acts synergistically with repressive histone modifications, including dimethylation or trimethylation of $\mathrm{H} 3-\mathrm{K}$ 9, to consolidate gene transcriptional silencing (22). However, it is not clearly understood how the formation of histone modifications may affect DNA methylation and which genes are involved with GC formation.

EFEMP1, also known as fibulin-3, is located on human chromosome $2 \mathrm{p} 16$, and is one of seven members of the fibulin gene family of extracellular glycoproteins. It contains 11 exons and encodes a 54-kDa protein. EFEMP1 regulates cell proliferation and cell-to-cell and cell-to-matrix communication, providing organization and stability to extracellular matrix structures (23). The precise mechanism underlying the role of EFEMP1 in the progression of tumors remains largely unknown. Hu et al (24) found that the overexpression of EFEMP1 inhibited glioma cell development and suppressed angiogenesis, vascular endothelial growth factor receptor A expression and cell proliferation. The EGF receptor level was reduced and AKT signaling activity attenuated following treatment with exogenous EFEMP1. Kim et al (25) reported that overexpression of EFEMP1 may inhibit non-small cell lung cancer cell invasion by downregulating cellular matrix metalloproteinase (MMP)-7 and MMP-2. However, the mechanism by which the expression of EFEMP1 was inhibited remains unclear. Frequent DNA methylation of the EFEMP1 promoter has been detected in lung cancer (5), hepatocellular carcinoma (6), prostate cancer (7), sporadic breast cancer (8) and colon tumors (26), but has not been reported in GC.

In the present study it was identified that EFEMP1 expression was significantly reduced in GC cell lines and tissues compared with normal gastric cells and tissues. It was demonstrated that EFEMP1 may function as a tumor suppressor in GC. In addition, two mechanisms underlying the decreased expression of EFEMP1 were identified, including DNA hypermethylation of the EFEMP1 promoter and hypermethylation of $\mathrm{H} 3-\mathrm{K} 9$ attached to the promoter.

In the present study, the EFEMP1 gene was hypermethylated in MKN45 and AGS cells, partially methylated in BGC823 cells, but not methylated in SGC7901 cells. The differential EFEMP1 expression and the methylation status of the gene between the four GC cell lines may be associated with the cell type. By contrast, aberrant methylation of EFEMP1 gene was also observed in primary gastric carcinomas. Hypermethylation of the EFEMP1 gene was detected in $29(64.44 \%)$ of the 45 primary gastric carcinomas, while methylation in non-malignant gastric tissues was only identified in 11 cases (24.44\%). Furthermore, it was demonstrated that GC with invasion depth at T3 and T4 had a notably higher methylation frequency than that with invasion depth at T1 and T2. Methylation frequency of EFEMP1 was also negatively correlated with tumor differentiation. These results suggest that the degree of malignancy of GC may be enhanced when the methylation frequency of EFEMP1 is high. These results are consistent with that of a study by Tong et al (26), who reported that aberrant methylation caused EFEMP1 downregulation in colorectal cancer, and EFEMP1 downregulation was correlated with lymph node metastasis, tumor stage and poor survival. These results are also supported by Sadr-Nabavi et al (8), who demonstrated that the level of EFEMP1 expression decreased in sporadic breast cancer due to its aberrant promoter methylation and was correlated with poor survival as an antagonist of angiogenesis. Yang et al (27) found EFEMP1 hypermethylation in 65/97 (67\%) endometrial carcinoma tissues compared with 4/40 (10\%) normal tissues. Their results demonstrated that the downregulation of EFEMP1 was associated with promoter hypermethylation.

Histone modification is another critical epigenetic process that facilitates the control of chromatin structure and gene regulation, which is associated with DNA methylation status in regulating gene expression. Unmethylated $\mathrm{CpG}$ islands are enriched in activated chromatin, but methylated DNA is associated with repressed chromatin (28). Using ChIP techniques in four GC cell lines, the present study demonstrated that H3-K9 trimethylation in the EFEMP1 promoter region was also closely associated with DNA methylation and acted as a marker of gene silencing. It was revealed that H3-K9 trimethylation of the EFEMP1 gene promoter correlated markedly with DNA methylation status. H3-K9 trimethylation levels in the EFEMP1 gene promoter were lower in the unmethylated GC cell lines (SGC7901) with high expression of EFEMP1; however, H3-K9 trimethylation levels in the EFEMP1 gene promoter were higher in the hypermethylated GC cell lines (MKN45, AGS and BGC823) with low expression of EFEMP1. In contrast to $\mathrm{H} 3-\mathrm{K} 9$ trimethylation, it was demonstrated that H3-K9 acetylation was inversely correlated with DNA methylation status.

To further examine the correlation between epigenetic alteration and EFEMP1 mRNA expression, four GC cell lines were treated with DAC and TSA. DAC, a pyrimidine analog with the 2'-deoxycytidine fifth carbon atom replaced by nitrogen, is able to form a complex with DNMT1 following binding to DNA during replication and subsequently inhibits transmethylation activity of this enzyme. TSA is an HDAC inhibitor and causes DNA histone hyperacetylation and induces p21 $1^{(\mathrm{WAF} 1 / \mathrm{CIPl})}$ gene expression (29). Consistent with previous studies $(30,31)$, DAC inhibited migration invasion and proliferation of MKN45 cells, but TSA had a weaker effect on the biological behavior of MKN45 cells. The present study also identified that DAC restored EFEMP1 expression in MKN45, AGS and BGC823 cells, which have low expression of EFEMP1 mRNA. In addition to its effect on DNA methylation, DAC reduced the level of $\mathrm{H} 3-\mathrm{K} 9$ trimethylation in the EFEMP1 promoter, but DAC had no significant effect on H3-K9 acetylation. TSA alone significantly increased H3-K9 acetylation but it did not restore EFEMP1 mRNA expression. A combination of DAC and TSA markedly increased Lys-9 acetylation and decreased Lys-9 methylation, and was most effective in restoring EFEMP1 gene expression in MKN45, AGS and BGC823 cells. However, in SGC7901 cells, which express EFEMP1 mRNA, treatment with DAC and TSA, alone or in combination, had no significant effect on the 
expression of EFEMP1. These results indicated that promoter DNA methylation and $\mathrm{H} 3-\mathrm{K} 9$ trimethylation, but not $\mathrm{H} 3-\mathrm{K} 9$ acetylation, are involved in the repression of EFEMP1 gene expression in human GC cells. It was also identified that DAC not only demethylated DNA promoters, but also altered existing histone $\mathrm{H} 3-\mathrm{K} 9$ trimethylation. DAC-induced changes in histone modifications were limited in the DNA hypermethylated cells (MKN45, AGS and BGC823). The mechanism underlying the modifications of histone methylation by DAC remain unclear. One simple possibility is that DNMTs, together with the methyl-CpG-binding protein MECP2, are able to recruit histone $\mathrm{H} 3-\mathrm{K} 9$-specific methyltransferases SUV39H1. Therefore, epigenetic information embodied in methylated residues flows from DNA to histone and back. DNMT and SUV39H1 form complexes to regulate EFEMP1 gene expression. The decreased expression of DNMT1 induced by DAC may then lead to histone demethylation via disruption of these silencing complexes (32). These results are in agreement with previous studies that the ability of DAC to reactivate the expression of DSC3 and MASPIN genes tracked closely with the reductions of $\mathrm{H} 3-\mathrm{K} 9$ methylation levels in their promoter regions (33). Therefore, DNA methylation and histone modification may function together to regulate gene expression $(34,35)$. In addition, as epigenetic alterations are reversible, they are considered useful therapeutic targets. Recently, DAC was demonstrated to synergize with progesterone therapy to inhibit endometrial cancer cell growth and invasion (36). These findings suggest that chemotherapeutic drugs combined with epigenetic agents may be potentially utilized for future cancer therapy.

In conclusion, the present study demonstrated that EFEMP1 was downregulated in GC, which was mainly caused by aberrant DNA methylation and histone $\mathrm{H} 3-\mathrm{K} 9$ trimethylation. DAC acts via epigenetic alterations to reactivate EFEMP1 expression. The mRNA expression of EFEMP1 gene and EFEMP1 methylation were associated with invasion and metastasis, which may be potential prognostic factors for GC. These findings provide a foundation for the role of EFEMP1 gene in GC and its potential as a biomarker for early diagnosis, and may lead to the identification of novel targets for pharmacological intervention. Therefore, further in vitro and in vivo studies are required to detect the function of EFEMP1 in the progression of GC.

\section{Acknowledgements}

The present study was supported in part by a grant from the National Natural Science Foundation of China (grant no. 30572162), the Foundation of Liaoning Province Science and Technology Plan Project (grant no. 2013225021) and the Higher Specialized Research Fund for Doctoral Program of Ministry of Education of China (grant no. 20102104110001).

\section{References}

1. Jemal A, Bray F, Center MM, et al: Global cancer statistics. CA Cancer J Clin 61: 69-90, 2011.

2. Allis CD, Berger SL, Cote J, et al: New nomenclature for chromatin-modifying enzymes. Cell 131: 633-636, 2007.

3. Cantone I and Fisher AG: Epigenetic programming and reprogramming during development. Nat Struct Mol Biol 20: 282-289, 2013.
4. Albig AR, Neil JR and Schiemann WP: Fibulins 3 and 5 antagonize tumor angiogenesis in vivo. Cancer Res 66: 2621-2629, 2006

5. Yue W, Dacic S, Sun Q, et al: Frequent inactivation of RAMP2, EFEMP1 and Dutt1 in lung cancer by promoter hypermethylation. Clin Cancer Res 13: 4336-4344, 2007.

6. Nomoto S, Kanda M, Okamura Y, et al: Epidermal growth factor-containing fibulin-like extracellular matrix protein 1 , EFEMP1, a novel tumor suppressor gene detected in hepatocellular carcinoma using double combination array analysis. Ann Surg Oncol 17: 923-932, 2010.

7. Kim YJ, Yoon HY, Kim SK, et al: EFEMP1 as a novel DNA methylation marker for prostate cancer: array-based DNA methylation and expression profiling. Clin Cancer Res 17: 4523-4530, 2011.

8. Sadr-Nabavi A, Ramser J, Volkmann J, et al: Decreased expression of angiogenesis antagonist EFEMP1 in sporadic breast cancer is caused by aberrant promoter methylation and points to an impact of EFEMP1 as molecular biomarker. Int J Cancer 124: 1727-1735, 2009.

9. Hwang CF, Chien CY, Huang SC, et al: Fibulin-3 is associated with tumour progression and a poor prognosis in nasopharyngeal carcinomas and inhibits cell migration and invasion via suppressed AKT activity. J Pathol 222: 367-379, 2010.

10. En-lin S, Sheng-guo C and Hua-qiao W: The expression of EFEMP1 in cervical carcinoma and its relationship with prognosis. Gynecol Oncol 117: 417-422, 2010.

11. Davidson B, Stavnes HT, Holth A, Chen X, Yang Y, et al: Gene expression signatures differentiate ovarian/peritoneal serous carcinoma from breast carcinoma in effusions. J Cell Mol Med 15: 535-544, 2011.

12. Seeliger H, Camaj P, Ischenko I, et al: EFEMP1 expression promotes in vivo tumor growth in human pancreatic adenocarcinoma. Mol Cancer Res 7: 189-198, 2009.

13. Fahrner, JA, Eguchi S, Herman JG and Baylin SB: Dependence of histone modifications and gene expression on DNA hypermethylation in cancer. Cancer Res 62: 7213-7218, 2002.

14. Cameron EE, Bachman KE, Myöhänen S, Herman JG and Baylin SB: Synergy of demethylation and histone deacetylase inhibition in the re-expression of genes silenced in cancer. Nat Genet 21: 103-107, 1999.

15. Kuo $\mathrm{MH}$ and Allis $\mathrm{CD}$ : In vivo cross-linking and immunoprecipitation for studying dynamic Protein:DNA associations in a chromatin environment. Methods 19: 425-433, 1999.

16. Wang R, Zhang YW and Chen LB: Aberrant promoter methylation of FBLN-3 gene and clinicopathological significance in non-small cell lung carcinoma. Lung Cancer 69: 239-244, 2010.

17. Sharma S, Kelly TK and Jones PA: Epigenetics in cancer. Carcinogenesis 31: 27-36, 2010.

18. Meng CF, Zhu XJ, Peng G and Dai DQ: Role of histone modifications and DNA methylation in the regulation of $\mathrm{O}^{6}$-methylguanine-DNA methyltransferase gene expression in human stomach cancer cells. Cancer Invest 28: 331-339, 2010.

19. Meng CF, Zhu XJ, Peng G and Dai DQ: Promoter histone H3 lysine 9 di-methylation is associated with DNA methylation and aberrant expression of p16 in gastric cancer cells. Oncol Rep 22: 1221-1227, 2009.

20. Chang X, Zhang S, Ma J, et al: Association of NDRG1 gene promoter methylation with reduced NDRG1 expression in gastric cancer cells and tissue specimens. Cell Biochem Biophys 66: 93-101, 2013

21. Grewal SI and Moazed D: Heterochromatin and epigenetic control of gene expression. Science 301: 798-802, 2003.

22. Peters AH, Mermoud JE, O'Carroll D, et al: Histone H3 lysine 9 methylation is an epigenetic imprint of facultative heterochromatin. Nat Genet 30: 77-80, 2002.

23. Zhang Y and Marmorstein LY: Focus on molecules: fibulin-3 (EFEMP1). Exp Eye Res 90: 374-375, 2010.

24. Hu Y, Pioli PD, Siegel E, et al: EFEMP1 suppresses malignant glioma growth and exerts its action within the tumor extracellular compartment. Mol Cancer 10: 123, 2011.

25. Kim EJ, Lee SY, Woo MK, et al: Fibulin-3 promoter methylation alters the invasive behavior of non-small cell lung cancer cell lines via MMP-7 and MMP-2 regulation. Int J Oncol 40: 402-408, 2012

26. Tong JD, Jiao NL, Wang YX, Zhang YW and Han F: Downregulation of fibulin-3 gene by promoter methylation in colorectal cancer predicts adverse prognosis. Neoplasma 58: 441-448, 2011. 
27. Yang T, Qiu H, Bao W, et al: Epigenetic inactivation of EFEMP1 is associated with tumor suppressive function in endometrial carcinoma. PLoS One 8: e67458, 2013.

28. Jaenisch R and Bird A: Epigenetic regulation of gene expression: how the genome integrates intrinsic and environmental signals. Nat Genet 33 (Suppl): 245-254, 2003.

29. Nawrocki ST, Carew JS, Douglas L, et al: Histone deacetylase inhibitors enhance lexatumumab-induced apoptosis via a p21Cip1-dependent decrease in survivin levels. Cancer Res 67: 6987-6994, 2007.

30. Chang X, Li Z, Ma J, et al: DNA methylation of NDRG2 in gastric cancer and its clinical significance. Dig Dis Sci 58: 715-723, 2013.

31. Zhi Y, Chen J, Zhang S, et al: Down-regulation of CXCL12 by DNA hypermethylation and its involvement in gastric cancer metastatic progression. Dig Dis Sci 57: 650-659, 2012.
32. Fuks F, Hurd PJ, Deplus R and Kouzarides T: The DNA methyltransferases associate with HP1 and the SUV39H1 histone methyltransferase. Nucleic Acids Res 31: 2305-2312, 2003.

33. Wozniak RJ, Klimecki WT, Lau SS, Feinstein Y and Futscher BW: 5-Aza-2'-deoxycytidine-mediated reductions in G9A histone methyltransferase and histone H3 K9 di-methylation levels are linked to tumor suppressor gene reactivation. Oncogene 26: 77-90, 2007.

34. Lin W and Dent SY: Functions of histone-modifying enzymes in development. Curr Opin Genet Dev 16: 137-142, 2006.

35. Martin $\mathrm{C}$ and Zhang Y: The diverse functions of histone lysine methylation. Nat Rev Mol Cell Biol 6: 838-849, 2005.

36. Hu Q, Yu L, Chen R, et al: 5-aza-2'-deoxycytidine improves the sensitivity of endometrial cancer cells to progesterone therapy. Int J Gynecol Cancer 22: 951-959, 2012. 